\title{
RESIDUOS DE $\beta$-LACTÁMICOS EN LECHE CRUDA Y FACTORES ASOCIADOS A SU PRESENTACIÓN
}

\section{B-LACTAM RESIDUES IN RAW MILK AND FACTORS ASSOCIATED WITH ITS PRESENTATION}

\author{
Juan Fernando Vásquez¹, Martha Olivera²
}

\begin{abstract}
${ }^{1}$ MV, M.Sc. Asistente Técnico Cooperativa Colanta Ltda. Grupo de investigación Biogénesis. Escuela de Medicina Veterinaria, Universidad de Antioquia. Autor para correspondencia: Carrera 75 № 65-87 oficina 46-202 Medellín, Colombia. E-mail: jufevaca@gmail.com 2MV, Dr.ScAgr. Docente Escuela de Medicina Veterinaria. Grupo de investigación Biogénesis. Universidad de Antioquia.
\end{abstract}

REV. U.D.C.A ACT. \& DIV. Cient. 15(1): 157 - 165, (2012)

\section{RESUMEN}

El Codex Alimentarius determina que la residualidad de antibióticos en leche debe ser nula por las consecuencias negativas para la salud humana (Codex Alimentarius Commission, 2011). Con el fin de aportar información sobre residuos $\beta$ lactámicos, los más usados en Colombia, en el control de infecciones en ganado lechero y para hacer una aproximación a los factores de riesgo asociados a la presencia de residuos, se analizaron las bases de datos de once plantas de acopio de leche cruda, de diferentes regiones del país. Se determinó la frecuencia de la residualidad de antibióticos, durante el 2010, y los factores de riesgo asociados, se buscaron en las encuestas aplicadas por parte de profesionales de los centros de acopio a los hatos positivos, durante el período 2007 a 2010. Las plantas estudiadas recibieron leche de 8.423 productores; de 738 millones de litros muestreados el 0,05\% de la leche fue positiva. Se analizaron 340 encuestas, en las que se indagó sobre el uso de antibióticos y los posibles factores de riesgo presentes para el no retiro de la leche y las posibles causas de no retiro de la leche. Los dos mayores factores de riesgo asociados a la residualidad fueron el uso de medicamentos sin prescripción médico veterinaria y la ausencia de registro de tratamientos. Adicionalmente, se asociaron al evento de positividad para residuos, el olvido en el retiro de la leche $(42,57 \%)$ y el tiempo de retiro incompleto (20,79\%). Los $\beta$ lactámicos más frecuentes fueron mezclas de cloxacilina-ampicilina (20\%), mezclas de penicilinas G $(15,2 \%)$ y, en menor proporción, cefalosporinas, amoxicilinas y penetamato. Las enfermedades asociadas a la positividad en residualidad fueron mastitis clínica (76\%) y cojeras (16\%).

Palabras clave: Antibióticos, inhibidores, mastitis, residuos en leche, salud pública.
SUMMARY

The Codex Alimentarius determines that residual antibiotics in milk should be zero due to the negative effects on human health. With the aim tocontribute to the information on residues of $\beta$ lactamics, the most common antimicrobials used in Colombia for the control infections in dairy cattle, and for making an approach to risks factors associated to the event of residues in milk we analyzed the data bases of eleven dairy collection facilities from different regions of the country. The frequency of residues was determined during 2010 and risk factors were searched in surveys applied by professionals belonging to the collection facilities to the herds appearing positive to residues during the period 2007 a 2010 . This Centers received milk from 8423 producers; out of the 738 millions litters sampled $0.05 \%$ were positive. 340 surveys were analyzed and the two major risk factors associated to residues were the use of medications without a professional prescription and the lack of medical histories. Other factors associated were forgetting the retirement $(42.57 \%)$ and incomplete retirement period (20.79\%) The $\boldsymbol{\beta}$-lactámicos most frequently found were mixtures of cloxaciline-ampiciline (20\%), mixtures of peniciline $\mathrm{G}(15.2 \%)$ and to a lesser extend cefalosporines, amoxicilines y penetamate. Diseases associated to positivity were clinical mastitis (76\%) and lameness (16\%).

Key words: Antibiotics, mastitis, residues in milk, public health.

\section{INTRODUCCIÓN}

La presencia de residuos de antibióticos y otros medicamentos en la leche es un tema de preponderancia en la salud pública actual, ya que tiene implicaciones en salud humana, 
por los fenómenos de resistencia a antibióticos, reacciones alérgicas hipersensibles (Bogialli \& Di Corcia, 2009) y toxicidad aguda o crónica (Doyle, 2006). En los procesos industriales, se dificultan los procesos de elaboración de quesos, de yogures y de otros derivados, que impliquen fermentación con cultivos bacterianos (Seymour et al. 1988; Van Schaik et al. 2002; Blowey \& Edmondson, 2010). A nivel comercial tienen implicaciones por la imposibilidad de vender leche o derivados con residuos, además de la imagen negativa de la opinión pública hacia el consumo de leche y sus derivados (McEwen et al. 1991). Las leyes y los principios que regulan la presencia de residuos de medicamentos y, en general, la inocuidad de los alimentos están consignados en el Codex Alimentarius. En Colombia, la norma que estipula los requisitos que debe cumplir la leche para el consumo humano es el decreto 616 de 2006 (Ministerio de Protección Social, 2006) y el 1880 de 2011 (Ministerio de Agricultura y Desarrollo Rural); Estados Unidos está regida por la Pasteurized Milk Ordinance PMO (US FOOD AND DRUG ADMINISTRATION FDA, 2007), mientras que para la Unión Europea es el reglamento \#2377/90; todas coinciden en que la cantidad de inhibidores en leche debe ser nula (McLaughlin, 2006).

El objetivo de este trabajo es generar información actualizada sobre la frecuencia de presentación de residuos de $\beta$-lactámicos en la leche cruda en Colombia, los factores de riesgo de su presentación, así como los factores de riesgo más probables, por las cuales, se entregó leche con residuos.

\section{MATERIALES Y MÉTODOS}

Tipo de estudio: Se realizó un estudio retrospectivo crosssectional, en el que los datos estudiados correspondían a los reportes del análisis de $\beta$-lactámicos en leche, realizados por una empresa colombiana de lácteos, en la totalidad de la leche enviada por 8.423 productores, durante el 2010.

Centros de acopio: Los centros de acopio analizados reciben leche de hatos localizados en diversos ecosistemas y con diferentes sistemas de producción. En las zonas de lechería especializada de trópico de altitud (>2000 m.s.n.m.), se encuentran las plantas de Funza, de San Pedro de los Milagros, de Santa Rosa de Osos y de Yarumal. Las plantas de Armenia, de Medellín y de Frontino tienen un área de influencia predominantemente de trópico medio (500-2000 m.s.n.m.), con sistemas de producción mixtos, en algunas zonas de lechería especializada y en otros de doble propósito y las plantas de Puerto Boyacá, de Barranquilla, de San Onofre y de Planeta Rica pertenecen a zonas de trópico bajo $(<500$ m.s.n.m.), donde predominan los sistemas de producción doble propósito (Figura 1).
Encuestas: Se analizaron las encuestas realizadas a los hatos que suministraron leche positiva, entre junio de 2007 y junio de 2010; estas encuestas fueron aplicadas por los Médicos Veterinarios del Departamento de Asistencia Técnica de la empresa acopiadora y, en ellas, se indagó sobre los factores de riesgo más probables de encontrar residuos antimicrobianos en leche, entre otras: enfermedades tratadas, antibióticos utilizados, normas de manejo de animales tratados, tiempo de retiro, disposición de la leche retirada, asistencia médico veterinaria.

Análisis estadístico: A partir de la información suministrada por las oficinas de control de calidad de las plantas, se calculó el volumen de leche positiva por planta/año (No. de litros positivos), número de tanques positivos (No. tanques de enfriamiento de leche en finca positivos) y porcentaje del volumen de leche positiva a $\beta$-lactámicos (volumen de leche positiva/volumen total captado por planta anualmente *100). Los datos, se sometieron a los estadísticos de Kolmogorov - Smirnov y de Shapiro - Wilk, comprobando que la distribución de los mismos cumplían los supuestos de normalidad. Ante esta situación, se procedió a determinar diferencias estadísticas en volumen y en porcentaje de leche positiva a $\beta$-lactámicos, entre plantas, mediante un ANAVA no paramétrico de Kruskal Wallis. Estas pruebas fueron procesadas, a través del programa estadístico SPSS ${ }^{\circledR}$ versión 17.0.

\section{RESULTADOS Y DISCUSIÓN}

Los resultados que se presentan a continuación son un reflejo de lo que sucede en producción de leche, en aquellos sitios donde se conserva la cadena de frío y donde los ganaderos son bonificados o penalizados, de acuerdo a la calidad de leche que entregan. Las grandes plantas acopiadoras de leche realizan pruebas en plataforma para determinar si tiene residuos antibióticos y penalizan a los productores recurrentes en esta práctica; sin embargo, autorizada por el decreto 1880 del Ministerio de Agricultura y Desarrollo Rural (2011), más de 17 millones de litros diarios de leche se comercializan crudos, sin pruebas de residualidad. Ejemplo de lo que puede ocurrir con la venta de leche cruda son los resultados de un estudio realizado en una cuenca lechera antioqueña, en donde los productores no tienen cadena de frío, se transporta la leche en cantinas y no están dentro de los programas de implementación de buenas prácticas ganaderas y se encontró un porcentaje de muestras positivas a $\beta$-lactámicos, que varió entre 4,6\% y 19,6\% (Reyes et al. 2010).

En Colombia, la producción de leche estimada durante 2009 fue de 5.760 millones de litros (Suárez \& Bazzani, 2010). El 12,82\% de la producción nacional (738.667.771 litros de leche refrigerada) fueron acopiados por las plantas analizadas en este estudio. Un total de 372.821 litros de le- 


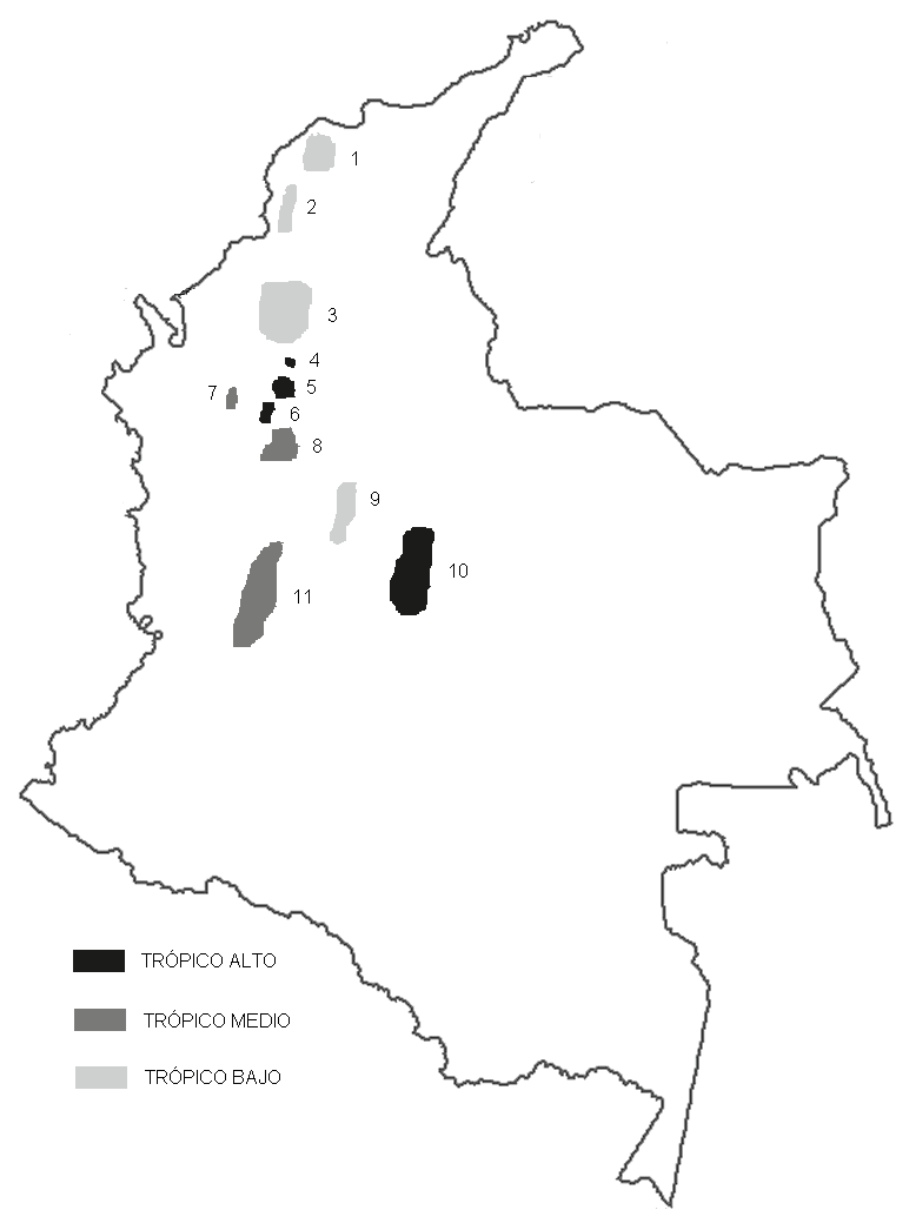

Figura 1. Áreas de influencia de los centros de acopio de leche en estudio y su zona de vida predominante. 1. Barranquilla, 2. San Onofre, 3. Planeta Rica, 4. Yarumal, 5. Santa Rosa, 6. San Pedro, 7. Frontino, 8. Medellín, 9. Puerto Boyacá, 10. Funza, 11. Armenia.

che provenientes de 733 productores resultaron positivos a $\beta$-lactámicos, lo que equivale al 0,05\% de la leche analizada. Del total de productores positivos, el 10,4\% reincidieron en entregar leche con residuos, a lo largo del año estudiado (2010). En su orden, las regiones cuyos tanques presentaron mayor frecuencia de residuos antimicrobianos correspondieron a las de sistemas de producción de lechería especializada de trópico alto y medio, localizadas en Antioquia (Santa Rosa de Osos, Yarumal, Medellín), viejo Caldas (Armenia) y Sabana de Bogotá (Funza), que oscilaron entre el 0,04 $0,10 \%$ de leche positiva (Tabla 1 ). En las regiones de trópico bajo de sistemas predominantemente de doble propósito, se presentó la menor incidencia, oscilando entre 0 y 0,01\%, de la leche acopiada. El análisis de varianza encontró que el porcentaje del volumen de leche positiva fue significativamente diferente $(\mathrm{P}<0.01)$ en, al menos, una de las plantas analizadas, además permitió determinar que el porcentaje de leche positiva a $\beta$-lactámicos durante el período de estudio fue también significativamente diferente $(P<0.01)$ en, al menos, uno de los centros de acopio; la prueba no paramétrica utilizada no permitió determinar cuáles en particular.

La frecuencia de resultados positivos a residuos $(0,05 \%)$ fue inferior a los encontrados para este tipo de antibióticos, en Perú, en la región del Callao, en donde de 40 muestras de leche cruda registraron $40 \%$ de muestras positivas a $\beta$-lactámicos (Guerrero et al. 2009) e inferiores a los reportados para Venezuela, en el estado Zulia, de 104 muestras de leche pasteurizada, de ocho marcas diferentes, encontraron el 0,96\% positiva a Penicilina G (Allara et al. 2002). En Paraná (Brasil), 59 de 151 muestras de leche pasteurizada contenían residuos de antimicrobiales, principalmente, neomicina, estreptomicina, dihidroestreptomicina y cloranfenicol (Bando et al. 2009); en México analizaron leche pasteurizada de cua- 
Tabla 1. Número de productores con resultados positivos a antibióticos $\beta$-lactámicos, en las diferentes regiones colombianas, durante 2010.

\begin{tabular}{|c|c|c|c|c|c|}
\hline $\begin{array}{l}\text { Zona de } \\
\text { producción }\end{array}$ & Planta & $\begin{array}{l}\mathrm{N}^{\circ} \text { productores } \\
\text { positivos }\end{array}$ & $\begin{array}{l}\text { Litros positivos a } \\
\text { antibióticos* }\end{array}$ & $\begin{array}{l}\text { Litros de leche } \\
\text { captada }\end{array}$ & $\begin{array}{l}\text { \% de leche } \\
\text { positiva* }\end{array}$ \\
\hline \multirow{4}{*}{$\begin{array}{l}\text { Trópico alto } \\
\text { (>2000 msnm) }\end{array}$} & Funza & 37 & 53.436 & 81.060 .043 & 0,07 \\
\hline & Yarumal & 103 & 28.923 & 47.169 .383 & 0,06 \\
\hline & Santa Rosa & 104 & 65.468 & 113.714 .523 & 0,06 \\
\hline & San Pedro & 327 & 112.094 & 282.190.819 & 0,04 \\
\hline \multirow{3}{*}{$\begin{array}{l}\text { Trópico medio } \\
\text { ( } 500-2000 \\
\text { msnm) }\end{array}$} & Armenia & 17 & 27.106 & 26.642 .296 & 0,10 \\
\hline & Frontino & 25 & 9.206 & 12.477 .941 & 0,07 \\
\hline & Medellín & 119 & 76.239 & 112.955 .796 & 0,07 \\
\hline \multirow{4}{*}{$\begin{array}{l}\text { Trópico bajo } \\
\text { (<500 msnm) }\end{array}$} & Pto. Boyacá & 1 & 349 & 6.315 .676 & 0,01 \\
\hline & Planeta Rica & 0 & 0 & 46.130 .167 & 0,00 \\
\hline & Barranquilla & 0 & 0 & 5.533 .013 & 0,00 \\
\hline & San Onofre & 0 & 0 & 4.478 .115 & 0,00 \\
\hline Total & & 733 & 372.821 & 738.667 .771 & 0,05 \\
\hline
\end{tabular}

"El análisis de varianza presentó diferencias significativas $(\mathrm{P}<0.01)$ para litros de leche positiva y porcentaje de leche positiva entre las plantas analizadas

tro industrias lecheras, hallando residuos de sulfonamidas, que oscilaron entre 44,7 y $58,3 \%$ de las muestras. Tres de 96 muestras excedieron los niveles de residuo máximo para sulfonamidas y una de 96 fue positiva para cloranfenicol (Gutiérrez et al. 2005); en otro estudio encontraron más del $50 \%$ de leche positiva a ampicilina, dicloxacilina, eritromicina, penicilina g y cloranfenicol (Ramírez et al. 2001) y en el estado Guerrero, el $18,6 \%$ de las muestras de leche cruda analizadas presentó residualidad (Camacho et al. 2010). En el caso de Colombia, entre tanto, se registró presencia de antibióticos, en el 25\% de leche cruda, en la ciudad de Montería (Máttar et al. 2009).

Estudios estadounidenses recientes reportan, entre 2008 y 2009, presencia de inhibidores, en 861 tanques, que contenían 14.578.704 kilos de leche cruda (NMDR, 2009). En el Estado de New York detectaron antibióticos en 3,9 por cada 1000 productores (Van Schaik et al. 2002). En la Unión Europea, el porcentaje de hatos positivos a antimicrobianos es menor al 0,5\% (Hillerton \& Berry, 2004). En Kenia, la positividad a antibióticos estuvo en $4 \%$, en un estudio (Ekuttan et al. 2007) y $21 \%$ en otro adelantado por Shitandi \& Sternesjo (2001), mientras que en Tanzania, el 4,5\% de las muestras de leche cruda tomadas fue positiva a residuos de antimicrobiales, en un estudio (Mdegela et al. 2009) y al 7,0\% de las muestras en otro (Kivaria et al. 2006), aunque para el 2000, la prevalencia de antibióticos llegó a ser del 36\% (Kurwijila et al. 2006). En Colombia, las sanciones para esta infracción no están reglamentadas. Dependiendo de la empresa acopiadora, las sanciones pueden ser desde ninguna hasta la suspensión del recibo de la leche, por períodos variables o de manera definitiva y penalizaciones en el precio de la leche. En Estados Unidos, la legislación es estricta: se sanciona con la suspensión parcial o permanente de licencia como productor de leche grado "A", el pago por parte del productor de la leche contaminada y de los costos generados en su descarte (Seymour et al. 1988; Sischo, 1996; US FOOD AND DRUG ADMINISTRATION FDA, 2007).

Este estudio muestra que la leche producida bajo sistemas de producción de lechería especializada es más propensa a enviar leche con residuos al acopiador que los hatos con sistemas doble propósito en Colombia, posiblemente, por una mayor prevalencia de mastitis, asociada a mayores producciones por vaca, con razas especializadas de producción lechera; en contraste, los sistemas doble propósito de trópico bajo, localizados en la costa Atlántica y Magdalena medio presentaron mucha menor positividad $(0-0,01 \%$ del total de la leche). Esto podría estar asociado a la menor utilización de antibióticos y algunas rutinas de manejo características del sistema, que pueden evitar el contacto de leche positiva con el tanque, tales como el aislamiento de animales tratados en corrales o potreros de enfermería y el darle toda la producción de leche de vacas en tratamiento a sus crías. Al- 
gunos estudios han reportado menor incidencia de mastitis en estos sistemas y lo han atribuido a la acción del ternero durante el mamado, por mejor extracción de leche residual, la presencia de enzimas bacteriostáticas presentes en la saliva en contacto con el pezón y el efecto de masaje en la ubre realizado por éste, durante la succión (Roldán et al. 2000).

Como se ve en la tabla 2, el análisis de las encuestas muestra que el olvido en el retiro de la leche es el primer factor de riesgo asociado a residuos en leche de $\beta$-lactámicos $(42,57 \%$ de los casos). Le siguen en importancia, el tiempo de retiro incompleto de los medicamentos (20,79\%), sea por desconocimiento de los tiempos de retiro o por omisión intencional de los mismos. La sobredosificación de antibióticos mostró residuos particularmente con los preparados farmacéuticos, basados en ceftiofur, los cuales, a dosis recomendadas no lo presentarían, pero a dosis mayores superan los límites máximos permitidos. También la vía inadecuada de aplicación, como la aplicación de productos de uso parenteral vía intramamaria (mezclas de penicilinas, por ejemplo) o lavados uterinos con antibióticos parenterales es causa de residuos en leche; en un caso, una finca entregó leche con residuos después de utilizar un antibiótico parenteral aplicado vía conjuntival.

En cuanto a los factores de riesgo que llevan a encontrar residuos en leche, los resultados de este trabajo concuerdan con los reportados en la literatura de países, como Reino Unido, Canadá y Estados Unidos: ausencia o mal manejo de registros de tratamiento, tiempo de retiro incompleto, falta de asistencia veterinaria, período de secado cortos en vacas tratadas con terapia antibiótica de vaca seca, accidentes u olvidos en la manipulación de leche de descarte, retiro de la leche de un solo cuarto en tratamientos intramamarios, fallas en la comunicación entre empleados del hato, trabajadores de medio tiempo, vacas recién compradas, desconocimiento de los tiempos de retiro de la leche, usos extraetiqueta de los productos y mala identificación de animales tratados. Es posible que la falta de rutina de realizar pruebas rápidas de detección de antibióticos en la finca también haya influido en que no se retire la leche el tiempo suficiente y se mezcle con la leche del tanque, como lo demuestran autores como Booth (1982), Kaneene \& Ahl (1987) y Sawant et al. (2005). Hatos grandes (Jayarao et al. 2004) en los que la identificación de los animales en tratamiento no es clara y hatos con altos recuentos de células somáticas en tanque tienden a ser más susceptibles, a presentar residuos antimicrobianos (Sargeant et al. 1998; Van Schaik et al. 2002, Reyes et al. 2010), ya que existe una asociación entre la salud de la ubre y el recuento de células.

Las causas más frecuentes por las que se encontraron residuos de $\beta$-lactámicos en la leche coinciden con las encon-

Tabla 2. Factores de riesgo más probables para que se presenten resultados positivos a antibióticos $\beta$-lactámicos en leche cruda de diferentes regiones colombianas $(n=340$ encuestas), durante el periodo comprendido entre junio de 2007 y junio de 2010.

\begin{tabular}{|l|c|c|c|}
\hline \multicolumn{1}{|c|}{ Causa } & Número de casos & $\begin{array}{c}\text { \% del total de } \\
\text { encuestas }\end{array}$ & $\begin{array}{c}\text { \% de causas } \\
\text { conocidas }\end{array}$ \\
\hline Desconocida & 138 & 40,59 & 42,57 \\
\hline Olvidó retirar leche & 86 & 25,29 & 20,79 \\
\hline Tiempo de retiro incompleto & 42 & 12,35 & 13,37 \\
\hline $\begin{array}{l}\text { Uso de droga de secado pocos días antes } \\
\text { del parto }\end{array}$ & 27 & 7,94 & 5,45 \\
\hline $\begin{array}{l}\text { Sobredosificación de antibióticos sin } \\
\text { modificar el tiempo de retiro }\end{array}$ & 11 & 3,24 & 4,95 \\
\hline $\begin{array}{l}\text { Se trató la vaca vía intramamaria y solo se } \\
\text { retiró la leche del cuarto afectado }\end{array}$ & 10 & 2,94 & 4,46 \\
\hline Vía inadecuada de aplicación & 9 & 2,65 & 4,46 \\
\hline Aplicación de antibióticos de uso humano & 9 & 2,65 & 2,48 \\
\hline Ordeño de animales nuevos en el hato & 5 & 1,47 & 1,49 \\
\hline Sabotaje & 3 & 0,88 & $\mathbf{1 0 0}$ \\
\hline
\end{tabular}


tradas en el 32, 15,8 y 3\%, de los casos en Estados Unidos (Booth, 1982); además, Blowey \& Edmonson (2010) reportan que el uso extraetiqueta de antimicrobianos fue el causal del $17 \%$ de los casos de residuos en leche. Este uso extraetiqueta puede aplicar en nuestro estudio con las causales de sobredosificación de antibióticos sin retiro, vía inadecuada de aplicación y de aplicación de antibióticos de uso humano. La suma de estos tres motivos equivale al $14,37 \%$ de las causas totales, lo cual, no difiere mucho de lo encontrado en este estudio norteamericano. Generalizando, varias de las causas reportadas por la literatura son asociadas a error humano, por olvido, por problemas de comunicación, por tratamientos mal realizados o por contaminación accidental del tanque con leche positiva (Booth, 1982; Blowey \& Edmondson, 2010).

Se demuestra el uso de antibióticos de aplicación en humanos, en nueve fincas, a las cuales, se les determinó que la leche contenía residuos y que habían usado los medicamentos en tratamientos intramamarios y parenterales; el producto más utilizado en esta infracción fue la ceftriazona, empleada en tratamiento de mastitis clínica, en el norte de Antioquia (Plantas de San Pedro de los Milagros, Santa Rosa de Osos y Yarumal). El uso de medicamentos de uso humano en medicina veterinaria, no aprobados por los servicios de salud, es una práctica indeseable que presenta problemas de positividad de antibióticos en leche, por desconocimiento de la farmacocinética del producto en la ubre bovina. Los resultados de la encuesta que indaga sobre normas de manejo tendientes a prevenir la presencia de inhibidores antimicrobianos en leche, se presentan en la tabla 3.

El porcentaje de fincas en que existe prescripción de antibióticos por un Médico Veterinario es similar $(33,91 \%)$ a lo reportado en Estados Unidos (32\%) (Sawant et al. 2005). Es importante anotar que, tanto el porcentaje de hatos que lle- va registros de tratamiento $(37,61 \%)$ como los que marcan los animales tratados (40\%), es muy inferior a lo reportado en estudios canadienses, donde el $47,87 \%$ de las fincas llevan registros de tratamiento y el $71,28 \%$ de los hatos positivos marcan animales (McEwen et al. 1991). La falta de registros ha sido reportada como la causa principal de presentación de residuos, en el $32 \%$ de hatos en Estados Unidos (Booth, 1982). Los bajos porcentajes de cumplimiento de estas normas tendientes a disminuir la presencia de $\beta$-lactámicos en leche son factores de riesgo latentes para la reincidencia en la presentación de positividad, por lo tanto, la labor de capacitación a los trabajadores de las fincas en estas prácticas es muy importante, así como la necesidad de que siempre sea un veterinario quien prescribe el tratamiento.

La frecuencia de aplicación de $\beta$-lactámicos en los 125 tratamientos posterior a los que se produjeron residuos en leche fue por vía intramamaria $(51,2 \%)$, en 16 casos $(12,8 \%)$ el producto se usó en tratamientos preventivos (en terapia de vaca seca) y $109(87,2 \%)$, en tratamientos curativos. El subgrupo de $\beta$-lactámicos con mayor frecuencia de detección de residuos en leche fueron las cefalosporinas $(46,4 \%)$. Como antibióticos genéricos, el más común en presentación fueron las mezclas de cloxacilina-ampicilina, con el $20 \%$ (Tabla 4). La vía de aplicación asociada a residuos en la leche coincide con los reportados en otros artículos (Booth \& Harding, 1986; McEwen et al. 1991), que encontraron que se debía, principalmente, a los tratamientos intramamarios, tanto en lactancia como en el secado, seguido por los tratamientos parenterales, intrauterinos y orales.

La enfermedad ampliamente asociada a presencia de $\beta$-lactámicos en leche fue mastitis, con 84 casos $(77,1 \%)$, seguido de cojeras, con 17 (15,6\%), hallazgos que concuerdan con los reportados por Seymour et al. (1988), 76 y $16 \%$ de los casos, respectivamente. En menor proporción, se en-

Tabla 3. Prácticas tendientes a prevenir presencia de inhibidores en leche realizadas en hatos positivos en Colombia (20072010).

\begin{tabular}{|l|c|}
\hline \multicolumn{1}{|c|}{ Práctica realizada } & \% DE REALIZACIÓN \\
\hline $\begin{array}{l}\text { Conocimiento de los tiempos de retiro de los medicamentos } \\
\text { que utiliza }\end{array}$ & 87,6 \\
\hline $\begin{array}{l}\text { Descarta la leche en recipientes marcados destinados para } \\
\text { este fin }\end{array}$ & 45,1 \\
\hline Marca y/o aísla los animales tratados & 40,0 \\
\hline Lleva registro de tratamientos y de retiro de leche & 37,6 \\
\hline Prescripción de antibióticos por médico veterinario & 33,9 \\
\hline
\end{tabular}


Tabla 4. Antibióticos $\beta$-lactámicos utilizados previo a la presentación de residuos en tanque ( $n=125$ encuestas).

\begin{tabular}{|l|c|c|}
\hline \multicolumn{1}{|c|}{$\beta$-lactámico utilizado } & Número de casos & \% \\
\hline Cloxacilina-Ampicilina & 25 & 15,2 \\
\hline Penicilina G & 19 & 13,6 \\
\hline Cefoperazona & 17 & 12,8 \\
\hline Penicilina-estreptomicina & 16 & 10,4 \\
\hline Ceftiofur & 13 & 7,2 \\
\hline Ceftriazona & 9 & 6,4 \\
\hline Cefapirina & 8 & 3,2 \\
\hline Cefalonium & 4 & 3,2 \\
\hline Cefquinoma & 4 & 2,4 \\
\hline Cefalexina & 3 & 1,6 \\
\hline Amoxicilina & 2 & 0,8 \\
\hline Amoxicilina-Ácido clavulánico & 1 & 0,8 \\
\hline Ampicilina & 1 & 0,8 \\
\hline Cloxacilina & 1 & 0,8 \\
\hline Penetamatoyohidrato & 1 & 0,8 \\
\hline Penicilina-kanamicina & 1 & 100 \\
\hline Total & 125 & \\
\hline
\end{tabular}

contró metritis, con tres casos $(2,7 \%)$, retención de placenta, con dos $(1,9 \%)$ y conjuntivitis, dermatitis y neumonía, con uno cada una $(2,7 \%)$.

Es importante resaltar que en Colombia aún es permitida la comercialización de leche cruda con mínimos controles estatales. Los presentes resultados dan una idea de lo que sucede con leche que es remitida fría a plantas acopiadoras, con programas de control de calidad y supervisión estatal, pero no son extrapolables a otras condiciones de producción y de comercialización. Lo que sí se puede concluir, a partir de los estudios, es que los esfuerzos en detección de antibióticos por parte del Estado y de la industria, ligado a una legislación seria y operativa y a capacitación de los ganaderos y operarios en la finca contribuyen de forma decisiva en la disminución de la problemática.

Se concluye que aún se entrega leche a las acopiadoras con residuos de antibióticos $(0,05 \%)$, aunque la legislación internacional determina que debe ser nula y que el mayor factor de riesgo para que suceda es el olvido de retirar la leche.

Agradecimientos: Los autores agradecen a los departamentos de Control Calidad y Asistencia técnica de la Cooperativa
Colanta, por permitir el acceso a sus bases de datos para el análisis de información y al MADR, por el apoyo a la formación de estudiantes de Maestría en Ciencias Animales, Fisiología de la Reproducción y la Lactancia, contrato 103200802526-3153. Conflictos de intereses: Los dos autores declaramos que no existe ningún conflicto de intereses que ponga en riesgo la validez de los resultados presentados. Financiación: Universidad de Antioquia.

\section{BIBLIOGRAFÍA}

1. ALLARA, M.; IZQUIERDO, P.; TORRES, G.; RODRÍGUEZ, B. 2002. Penicilina $G$ en leche pasteurizada producida en el estado Zulia - Venezuela. Rev. Científica. 12:683-687.

2. BANDO, E.; OLIVEIRA, R.C.; FERREIRA, G.M.; MACHINSKI, M. Jr. 2009. Occurrence of antimicrobial residues in pasteurized milk commercialized in the state of Paraná, Brazil. J. Food Prot. 72:911-914.

3. BLOWEY, R.; EDMONDSON, P. 2010. Mastitis Control in Dairy Herds. 2a ed. Londres. CAB International. 239p. 
4. BOGIALLI, S.; DI CORCIA, A. 2009. Recent applications of liquid chromatography-mass spectrometry to residue analysis of antimicrobials in food of animal origin. Anal. Bioanal. Chem. 395:947-966.

5. BOOTH, J.M. 1982. Antibiotic residues in milk. Practice. 4:100-109.

6. BOOTH, J.M.; HARDING, P. 1986. Testing for antibiotic residues in milk. Vet. Record.119:565-569.

7. CAMACHO, L.; CIPRIANO, M.; CRUZ, B.; GUTIÉRREZ, I.; HERNÁNDEZ, P.; PEÑALOZA, I.; NAMBO, O. 2010. Residuos de antibióticos en leche cruda comercializada en la región Tierra Caliente, de Guerrero, México. REDVET- 11:2.

8. CODEX ALIMENTARIUS. 2011. Residuos de Medicamentos Veterinarios en los alimentos. Disponible desde Internet en: http://www. codexalimentarius.net/vetdrugs/data/vetdrugs/index. html\#I (con acceso 26/02/2011).

9. DOYLE, M.E. 2006. Veterinary drug residues in processed meats - potential health risk. Food Research Institute (FRI Briefings). Disponible desde (nternet en: http:// fri.wisc.edu/docs/pdf/FRIBrief_VetDrgRes.pdf (con acceso 28/12/2011).

10. EKUTTAN, C.E.; KANG'ETHE, E.K.; KIMANI, V.N.; RANDOLPH, T.F.; 2007. Investigation on the prevalence of antimicrobial residues in milk obtained from urban smallholder dairy and non-dairy farming households in Dagoretti Division, Nairobi, Kenya. East Afr. Med. J. 84:87-91.

11. GUERRERO, D.; MOTTA, R.; GAMARRA, G.; BENAVIDES, E.; ROQUE, M.; SALAZAR, M. 2009. Detección de residuos de antibióticos $\beta$-lactámicos y tetraciclinas en leche cruda comercializada en El Callao. Ciencia e Invest. 12:79-82.

12. GUTIÉRREZ, R.; NOA, M.; DÍAZ, G.; VEGA Y LEÓN, S.; GONZÁLEZ, M.; PRADO, G. 2005. Determination of the presence of 10 antimicrobial residues in mexican pasteurized milk. INCI. 30:291-294.

13. HILLERTON, J.E.; BERRY, E.A. 2004. Quality of the milk supply: European regulations versus practice. NMC Annual Meeting Proceedings 2004. Compton, U.K. Disponible desde Internet en: http://www. nmconline.org/articles/qualityeuro.pdf (con acceso 28/12/2011).

14. JAYARAO, B.M.; PILLAI, S.R.; SAWANT, A.A.;
WOLFGANG, D.R.; HEGDE, N.V. 2004. Guidelines for monitoring bulk tank milk somatic cell and bacterial counts. J. Dairy Sci. 87:3561-3573.

15. KANEENE, J.B.; AHL, S. 1987. Drug residues in dairy cattle industry: epidemiological evaluation of factors influencing their occurrence. J. Dairy Sci. 70:21762180.

16. KIVARIA, F.M.; NOORDHUIZEN, J.P.; KAPAGA, A.M. 2006. Evaluation of the hygienic quality and associated public health hazards of raw milk marketed by smallholder dairy producers in the Dar es Salaam region, Tanzania. Trop Anim Health Prod. 38:185-194.

17. KURWIJILA, L.R.; OMORE, A.; STAAL, S.; MDOE, N.S. 2006. Investigation of the risk of exposure to antimicrobial residues present in marketed milk in Tanzania. J. Food Prot. 69:2487-2492.

18. MÁTTAR, S.; CALDERÓN, A.; SOTELO, D.; SIERRA, M.; TORDECILLA, G. 2009. Detección de Antibióticos en Leches: Un Problema de Salud Pública. Rev. Salud Pública. 11:579-590.

19. MCEWEN, S.; BLACK, W.; MEEK, A. 1991. Antibiotic residue prevention methods, farm management, and occurrence of antibiotic residues in milk. J. Dairy Sci. $74: 2128-2137$.

20. McLAUGHLiN, F. 2006. A brief comparison of United States and European Union standards for fluid dairy production. Michigan State University. Disponible desde Internet en: http://www.iflr.msu.edu/ BookStudentPapers_files/A_Brief_Comparison_of United_States_and_European_Union_Standards_ for_Fluid_Dairy_Prōucts.pdf ${ }^{-}$(con acceso 30/11/2011).

21. MDEGELA, R.H.; RYOBA, R.; KARIMURIBO, E.D.; PHIRI, E.J.; LOKEN, T.; REKSEN, O.; MTENGETI, E.; URIO, N.A. 2009. Prevalence of clinical and subclinical mastitis and quality of milk on smallholder dairy farms in Tanzania. J. S. Afr. Vet. Assoc. 80:163-168.

22. MINISTERIO DE AGRICULTURA Y DESARROLLO RURAL. 2011. Decreto 1880 de 2011. Disponible desde Internet en: www.minagricultura.gov.co/ archivos/decreto_leche_cruda.pdf (con acceso 30/11/2011).

23. MINISTERIO DE PROTECCIÓN SOCIAL. 2006. Decreto número 616 de 2006. Disponible desde Internet en: http://www.agronet.gov.co/www/docs 
agronet/2006103010449_decreto_616_28_02_06. pdf (con acceso 28/12/2011).

24. NMDR (NATIONAL MILK DRUG RESIDUE DATA BASE FISCAL YEAR). 2009. Annual Report October 1, 2008 - September 30 GLH Inc., Falls Church, VA, 2010. Disponible desde Internet en: http://www. kandc-sbcc.com/nmdrd/fy-09.pdf (con acceso 28/12/2011).

25. RAMÍREZ, A.; GUTIÉRREZ, R.; GONZÁLEZ, C.; ESCOBAR, I.; CASTRO, G.; DÍAZ, G.; NOA, M. 2001. Detección de antibióticos en leche comercializada en la ciudad de México. Rev. Salud Anim. 23:37-41.

26. REYES, J.; VILLAR, D.; OLIVERA, M. 2010. Evaluación de residuos de antimicrobianos por la prueba Delvotest, en una cuenca lechera de Antioquia con alto índice de mastitis subclínica. REDVET. 11:121009.

27. ROLDÁN, A.; PERDOMO, P.; SÁNCHEZ, H.; RAMÍREZ, M. 2000. Tecnificación del sistema de producción ganadera de doble propósito en el trópico andino colombiano: amamantamiento restringido. Livestock Res. Rural Devel. 12:2-4.

28. SARGEANT, J.; SCHUKKEN, Y.; LESLIE, K. 1998. Ontario Bulk Milk Somatic Cell Count Reduction Program: Progress and Outlook. J. Dairy Sci. 81:1545-1554.

29. SAWANT, A.; SORDILLO, L.; JAYARAO, B. 2005. A Survey on Antibiotic usage in dairy herds in Pennsylvania. J. Dairy Sci. 88:2991-2999.
30. SEYMOUR, E.; JONES, G.; McGILLIARD, M. 1988. Persistence of residues in milk following antibiotic treatment of dairy cattle. J. Dairy Sci. 71:2292-2296.

31. SHITANDI, A.; STERNESJO, A. 2001. Detection of antimicrobial drug residues in kenyan milk. J. Food Safety. 21:205-214.

32. SISCHO, W. 1996. Symposium: drug residue avoidance: the issue of testing quality milk and tests for antibiotic residues. J. Dairy Sci. 79:1065-1073.

33. SUÁREZ, R.; BAZZANI, A. 2010. Coyuntura de la leche en Colombia - Balance del año 2009. ANALAC. Área de estudios económicos; Disponible desde Internet en: http://establo.info/Documentos/COYUNTURA\%20 DE\%20LA\%20LECHE\%20EN\%20COLOMBIA\%20 Mz29.pdf (con acceso 28/12/2011).

34. US FOOD AND DRUG ADMINISTRATION - FDA. 2007. Center for Food Safety and Applied Nutrition. Grade "A" pasteurized milk ordinance: 2007 revision. Disponible desde Internet en: http://www.fda.gov/Food/ FoodSafety/Product-SpecificInformation/MilkSafety/ IConferenceonInterstateMilkShipmentsNCIMSModelDocuments/PasteurizedMilkOrdinance2007/default.htm (con acceso 28/12/2011).

35. VAN SCHAIK, G.; LOTEM, M.; SCHUKKEN, Y.H. 2002. Trends in somatic cell counts, bacterial counts, and antibiotic residue violations in New York state during 1999-2000. J. Dairy Sci. 85:782-789.

Recibido: Enero 2 de 2012

Aceptado: Abril 19 de 2012 